\title{
ON COMPOSITIONS OF THE LOOP AND SUSPENSION FUNCTORS
}

\author{
M. H. EGGAR
}

(Communicated by Thomas Goodwillie)

\begin{abstract}
The problem studied is whether, from knowledge of the homotopy

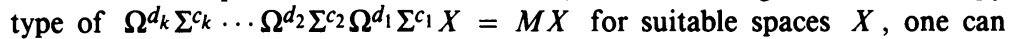
recover the nonnegative integers $c_{1}, d_{1}, \ldots, c_{k}, d_{k}$. The Betti numbers of $X$ and $c_{1}, d_{1}, \ldots, c_{k}, d_{k}$ do determine the $i$ th Betti number of $M X$, but even for small $k, i$ and for $X$ a sphere (say) the answer is a complicated one, since it depends on parities and graded Witt numbers depending on graded Witt numbers. It is shown that $k$ can be found and that $c_{i}, d_{j}$ can always be determined up to finitely many possibilities and usually uniquely.
\end{abstract}

In this paper we work in the homotopy category of pointed spaces which have the homotopy type of countable CW-complexes. The reduced suspension functor and its right adjoint, the loop functor, are functors from this category to itself, and many further functors can be generated using composition, product, smash product, and wedge sum. Results in classical homotopy theory (e.g., the Hilton-Milnor theorem) relate certain functors so formed, but the algebraic structure of the set of functors is not known.

This paper investigates a part of this problem. Let $\mathscr{M}$ denote the free monoid of reduced words in two indeterminates $\Sigma, \Omega$. The typical element $M$ of $\mathscr{M}$ is

$$
M=\Sigma^{A_{k+1}} \Omega^{B_{k}} \Sigma^{A_{k}} \ldots \Omega^{B_{2}} \Sigma^{A_{2}} \Omega^{B_{1}} \Sigma^{A_{1}},
$$

where $k, A_{1}, A_{k+1}$ are nonnegative integers and $B_{1}, A_{2}, B_{2}, \ldots, A_{k}, B_{k}$ are positive integers. We shall call a word $M$ positive if $A_{1}, B_{1}-1, A_{2}-1, B_{2}-1$, $\ldots, A_{k}-1, B_{k}-1, A_{k+1}$ are all positive integers. Each word $M$ determines a functor, namely, the corresponding composition of the reduced suspension $\Sigma$ and the loop functor $\Omega$. It is a natural question to ask whether different words give rise to different functors.

One can find infinitely many different words $M_{i} \in \mathscr{M}$ such that, for infinitely many noncontractible spaces $X, M_{i} X$ is homotopically equivalent to $M_{j} X$ for all $i, j$. In contrast we prove here

Theorem. Given $M \in \mathscr{M}$ there are at most finitely many $N \in \mathscr{M}$ such that, for all spaces $X, M X$ is homotopically equivalent to $N X$. If $M$ is positive, then $N=M$ is the only possibility.

Received by the editors July 22, 1992 and, in revised form, May 1, 1993.

1991 Mathematics Subject Classification. Primary 55P35; Secondary 55P62, 17 B70.

Key words and phrases. Loop, suspension, homotopy equivalence, rational homotopy theory. 
The task is to find an invariant that can be calculated in sufficient generality to deduce the theorem. We use rational homotopy. In $\S 1$ the requisite algebraic topology is assembled. Thereafter, the work is purely combinatorial. In $\S 2$ the combinatorial problem is stated (independently of the topology) and the finiteness in the theorem is established. In $\S 3$ the uniqueness result is proved.

It is reasonable to conjecture that in the theorem uniqueness holds for all $M$. It would be interesting if another invariant of algebraic topology or a more sophisticated combinatorial analysis of rational homotopy could establish the full conjecture. David Anick [1] has recently investigated the complexity of computing rational homotopy groups and shown the general problem to be "\# $P$ hard" (i.e., in a class of problems high on the computer scientists' complexity scale and believed to require more steps than a polynomial function of the (coded) input). This raises the philosophical question of whether there are conjectures similar to the above that are actually impossible to resolve by rational homotopy, because the general computation required is intractible in the necessary generality.

\section{The RATIONAL HOMOLOGY OF $M X$}

To calculate the rational homology $H_{*}(M X)$ we rewrite $(0.1)$ in the form

$$
M=\Sigma^{a_{k+1}} \Omega^{b_{k}}(\Omega \Sigma) \Sigma^{a_{k}} \ldots \Omega^{b_{2}}(\Omega \Sigma) \Sigma^{a_{2}} \Omega^{b_{1}}(\Omega \Sigma) \Sigma^{a_{1}},
$$

where $a_{k+1}=A_{k+1}$ and, for $1 \leq i \leq k, a_{i}=A_{i}-1$ and $b_{i}=B_{i}-1$. We shail suppose $X$ is a sufficiently connected space $\left(\left(b_{1}+\cdots+b_{k}\right)\right.$-connected suffices) and is a suspended space if $A_{1}=0$. The rational homology $H_{*}(M X)$ can now be computed using the three basic cases below.

Suppose $Y$ is a space for which $H=H_{*} Y$ and the subspace $P_{Y}$ of primitives in $H$ with respect to $\left(\Delta_{Y}\right)_{*}$ are known, where $\Delta_{Y}: Y \rightarrow Y \times Y$ is the diagonal map. We denote $\operatorname{rank} H_{i} Y$ by $\lambda_{i}(Y)$ and the (formal) Poincare series $\sum_{i=0}^{\infty} \lambda_{i}(Y) t^{i}$ for $Y$ by $\lambda(Y, t)$.

Case 1. If $Z=\Sigma^{a} Y$ then the Poincaré series $\lambda_{Z}(t)$ for $Z$ is given by

$$
\lambda(Z, t)=1+t^{a}(\lambda(Y, t)-1) .
$$

The subspace of primitives $P_{Z}$ in $H_{*} Z$ is $H_{*} Z$ if $a>0$ (and is $P_{Y}$ if $a=0$ ).

Case 2. If $Z=\Omega \Sigma Y$ then $H_{*} Z$ is the free associative graded tensor algebra on $H$, and hence formally,

$$
\begin{aligned}
\lambda(Z, t)= & 1+(\lambda(Y, t)-1)+(\lambda(Y, t)-1)^{2} \\
& +\cdots+(\lambda(Y ; t)-1)^{m}+\cdots .
\end{aligned}
$$

Furthermore, using the reduced product space model [2] for $Z$ one can describe $\left(\Delta_{Z}\right)_{*}$ completely in terms of $\left(\Delta_{Y}\right)_{*}$ and $i_{*}$, where $i: Y \rightarrow Z$ denotes the canonical inclusion. The subspace of primitives $P_{Z}$ of $H_{*} Z$ is isomorphic to the free graded Lie algebra on $H$. (We remark that $i_{*} H$ will, however, in general not be contained in $P_{Z}$; indeed, $i_{*} H \cap P_{Z}=i_{*}\left(P_{Y}\right)$.) The rank of $P_{Z}$ in dimension $n$ will be denoted by $\kappa_{n}(Z)$. The graded Witt formula giving $\kappa_{n}(Z)$ in terms of the coefficients $\lambda_{j}(Y)$ of $\lambda(Y, t)$ can be deduced from (4.5) 
in [3] or by generalizing [4] and is

$$
\kappa_{i}(Z)=\sum_{d \mid i}(-1)^{i+d} \mu\left(\frac{i}{d}\right) \frac{d}{i} \sum_{\substack{\beta_{j} \geq 0 \\ \sum_{j} \beta_{j}=d}} \frac{\left(\beta_{1}+\beta_{2}+\cdots\right) !}{\beta_{1} ! \beta_{2} ! \cdots} \frac{\lambda_{1}(Y)^{\beta_{1}} \lambda_{2}(Y)^{\beta_{2}} \cdots}{\left(\beta_{1}+\beta_{2}+\cdots\right)} .
$$

Here $\mu$ is the Möbius function.

Case 3. If $Z=\Omega^{b}(\Omega \Sigma Y)$, where $b>0$, then $H_{*} Z$ is the commutative graded tensor algebra on $P_{Z}$, since all Samelson products in $\pi_{*} Z$ vanish. The Poincaré series for $P_{Z}$ is

$$
\kappa(Z, t)=1+t^{-b} \sum_{i=1}^{\infty} \kappa_{i}(\Omega \Sigma Y) t^{i}
$$

and hence

$$
\lambda(Z, t)=\prod_{i \text { odd }}\left(1+t^{i}\right)^{\kappa_{i}} \prod_{i \text { even }}\left(1-t^{i}\right)^{-\kappa_{i}},
$$

where

$$
\kappa_{i}=\kappa_{i}(Z)=\kappa_{i+b}(\Omega \Sigma Y) .
$$

Clearly $H_{i}(M X)$ depends only on $M$ and $H_{*} X$, but the calculations become very complicated, even for small $k, i$ and for $X$ a sphere, say, as the ranks depend on parities and Witt numbers depending on Witt numbers.

\section{THE COMBINATORIAL PROBLEM}

For the sake of clarity it is worth extracting from the algebraic/topological context of $\S 1$ the combinatorial situation that we now study. For each $M \in \mathscr{M}$ as in $(0.1)$ we consider any polynomial $\lambda(t)$ with nonnegative integer coefficients and such that $\lambda(t)-1$ is divisible by $t^{\mu}$ where $\mu=-k+B_{1}+B_{2}+\cdots+B_{k}$. We now define two power series $\lambda(M, t)=1+\sum_{i>0} \lambda_{i}(M) t^{i}, \kappa(M, t)=1+$ $\sum_{i>0} \kappa_{i}(M) t^{i}$ by writing $M$ as in (1.1) and iteratively using the following cases.

Case 0 . For the empty word $I$ take $\lambda(I, t)=\kappa(I, t)=\lambda(t)$. (The empty word is the identity element of the monoid $\mathscr{M}$, i.e., the case $k=0, A_{1}=0$ in $(0.1)$.)

Case 1. If $Z=\Sigma^{a} Y$, where $Y \in \mathscr{M}$ and $a \geq 1$, then $\lambda(Z, t)$ and $\kappa(Z, t)$ are equal and given by (1.2).

Case 2. If $Z=\Omega \Sigma Y$, where $Y \in \mathscr{M}$, then $\lambda(Z, t)$ and $\kappa(Z, t)$ are given by (1.3) and (1.4), respectively.

Case 3. If $Z=\Omega^{b}(\Omega \Sigma Y)$, where $Y \in \mathscr{M}$ and $b \geq 1$, then $\kappa(Z, t)$ and $\lambda(Z, t)$ are given by (1.5) and (1.6), respectively.

The problem is to endeavor to recover $M$ by choosing $\lambda(t)$ and $i$ suitably and observing $\lambda_{i}(M)$ and $\kappa_{i}(M)$. In this section we shall show how to determine $M$ up to finitely many possibilities.

Notation. Let $n$ be a positive integer. We will often need to consider the integral multiple of $n$ that lies "closest" to another positive integer $i$. To resolve the 
ambiguous cases we adopt the convention of taking this multiple to be $\left[\frac{i}{n}+\frac{1}{2}\right] n$. Furthermore, we write $i \sim j$ if $\left[\frac{i}{n}+\frac{1}{2}\right]=\left[\frac{j}{n}+\frac{1}{2}\right]$. For $M$ as in (0.1) we define $\|M\|$ to be $2 k-1+\sum A_{j}+\sum B_{j}$.

Henceforth, we shall always choose the polynomial $\lambda(t)$ so that $\lambda_{i}$ is zero unless $i$ lies close to an integral multiple of $n$. The first lemma confirms the "obvious" fact that for $n$ sufficiently large the early values of $i$ for which $\lambda_{i}(M)$ is nonzero all lie close to integral multiples of $n$. More precisely, given $R, u \geq 0$, suppose that $\lambda_{i}=0$ if $\left|i-\left[\frac{i}{n}+\frac{1}{2}\right] n\right|>u$ and suppose that $\lambda_{i} \leq R$ for all $i$.

Lemma 1. Let $K, L$ be positive integers. Fix $u \geq 0$. There exist numbers $C(K, L, R), D(K, L), N(K, L)$ such that, whenever $n>N(K, L)$ and $M$ is any word satisfying $\|M\|<L$, then for each positive integer $i$ at least one of the following conditions is satisfied:

(i) $i>K n+\frac{n}{2}$,

(ii) $\lambda_{i}(M)=0$,

(iii) $\lambda_{i}(M)<C(K, L, R)$ and $\left|i-\left[\frac{i}{n}+\frac{1}{2}\right] n\right|<D(K, L)$.

The proof of Lemma 1 is unenlightening and can be found in the appendix. (We shall only use the case $u=0$ in this paper, but the proof of the lemma is not much simpler for the special case and so is given in general.) Henceforth, we shall always assume that $n$ has been chosen sufficiently large so that, for the $R, K, L, u$ being considered, $D(K, L)$ and $C(K, L, R)$ are small in comparison with $n$.

We now investigate $\lambda_{i}(M)$ for $i<K n+\frac{n}{2}$ in more detail. To facilitate the statements of Propositions 2 and 5, it is convenient to introduce

Notation. A $2 k$-tuple $\mathbf{x}=\left(x_{1}, \ldots, x_{2 k}\right)$ of positive integers is said to be $d e$ scending if $x_{i} \leq x_{i-1}$ for $i=2,3, \ldots, 2 k$. The $2 k$-tuple $\mathbf{x}$ is said to be cascading if $x_{i-1}$ is divisible by $x_{i}$ for $i=2,3, \ldots, 2 k$. For $M$ as in (1.1) and $\mathbf{x}$ a $2 k$-tuple we define the integer $S(\mathbf{x})$ by

$$
S(\mathbf{x})=x_{1} a_{1}-x_{2} b_{1}+x_{3} a_{2}-x_{4} b_{2}+\cdots-x_{2 k} b_{k}+a_{k+1} \text {. }
$$

Proposition 2. Let $M$ be a word satisfying $\|M\|<L$, and take $\lambda(t)=1+r t^{n}$. If $i \sim j n$, where $j \leq K$ and $n>N(K, L)$, then $\lambda_{i}(M)=0$ unless $i$ has the form $i=j n+S(\mathbf{x})$ for some descending $2 k$-tuple $\mathbf{x}$ with $x_{1}=j$. In particular:

(i) For $j=1, \lambda_{i}(M)=r$ when $i=n+a_{1}-b_{1}+\cdots+a_{k}-b_{k}+a_{k+1}$ (and $\lambda_{i}(M)$ is zero otherwise).

(ii) For $j=2, \sum_{i \sim 2 n} \lambda_{i}(M) t^{i}=\sum_{\mathbf{x}} c(\mathbf{x}) t^{2 n+S(\mathbf{x})}$, where the summation is taken over all descending $2 k$-tuples $\mathbf{x}$ with $x_{1}=2$.

The coefficient $c(\mathbf{x})$ is $\left(\begin{array}{l}r \\ 2\end{array}\right)$ if $\mathbf{x}=(2,2, \ldots, 2,1, \ldots, 1)$ has exactly $2 j$ entries 2 and $n+a_{1}-b_{1}+a_{2}-b_{2}+\cdots+a_{j}-b_{j}$ is odd, or if $\mathbf{x}$ has exactly $(2 j-1)$ entries 2 and $n+a_{1}-b_{1}+\cdots+a_{j}$ is even. Otherwise $c(\mathbf{x})=\left(\begin{array}{c}r+1 \\ 2\end{array}\right)$.

A proof of this proposition by induction on $L$ is given in the appendix. We use the proposition to derive information about $M$ from observations of $\lambda_{i}(M)$ for $i \sim n$ and $i \sim 2 n$. From (i) we deduce the value of $C$, where

$$
C=a_{1}-b_{1}+a_{2}-b_{2}+\cdots+a_{k}-b_{k}+a_{k+1} \text {. }
$$


Next we observe from (ii) that, for fixed $M, r, \sum_{i \sim 2 n} \lambda_{i}(M)$ depends only on the parity of $n$. Furthermore, $c(\mathbf{x})$ is $\left(\begin{array}{l}r \\ 2\end{array}\right)$ (respectively, $\left(\begin{array}{c}r+1 \\ 2\end{array}\right)$ ) when $n$ is even if and only if $c(\mathbf{x})$ is $\left(\begin{array}{c}r+1 \\ 2\end{array}\right)$ (respectively, $\left.\left(\begin{array}{c}r \\ 2\end{array}\right)\right)$ when $n$ is odd. Thus if the sum $\sum_{i \sim 2 n} \lambda_{i}(M)$ is observed when $n$ is even, and then when $n$ is odd, the total of the two sums will be $2 k r^{2}$, since there are $2 k$ descending $2 k$-tuples $\mathbf{x}$ with $x_{1}=2$. We can thus deduce the value of $k$. Finally we show how to deduce the unordered sets

$$
\left\{a_{1}-b_{1}, a_{1}-b_{1}+a_{2}-b_{2}, \ldots, a_{1}-b_{1}+a_{2}-b_{2}+\cdots+a_{k}-b_{k}\right\}
$$

and

$$
\left\{a_{1}, a_{1}-b_{1}+a_{2}, \ldots, a_{1}-b_{1}+\cdots+a_{k}\right\} .
$$

The union of these two unordered sets is precisely the set of values of $S(\mathbf{x})-$ $C$ as $\mathbf{x}$ runs over all descending $2 k$-tuples with $x_{1}=2$. Choose $r>4 k$. Then $w\left(\begin{array}{l}r \\ 2\end{array}\right)$ is not a multiple of $\left(\begin{array}{c}r+1 \\ 2\end{array}\right)$ for $1 \leq w \leq 2 k$. Choose $n$ to be even, and observe the values of $i$ and $\lambda_{i}(M)$ such that $\lambda_{i}(M)$ is nonzero, $i \sim 2 n$, and $i$ has the same parity as $C$. Then from Proposition 2(ii) we can deduce the even numbers (with multiplicities) in (2.2) and also the even numbers in (2.3), since the former each correspond to an $\mathbf{x}$ contributing $\left(\begin{array}{c}r+1 \\ 2\end{array}\right)$ to $\lambda_{i}(M)$ and the latter to an $\mathbf{x}$ contributing $\left(\begin{array}{l}r \\ 2\end{array}\right)$ to $\lambda_{i}(M)$. Likewise from those $i$ with opposite parity to $C$ one can read off the odd numbers (with multiplicities) in the unordered sets (2.2) and (2.3). There are only finitely many ways of ordering the elements in the unordered sets (2.2) and (2.3), and elementary algebra uniquely determines $a_{1}, b_{1}, \ldots, a_{k}, b_{k}$ once the ordering is fixed. Hence, using (2.1) to find $a_{k+1}$, we see that there are only finitely many possibilities for $M$.

The conclusions obtained above are summarized by

Corollary 3. The values of $k,(2.1)$, and the unordered sets (2.2) and (2.3) may be deduced from observations of $\lambda_{i}(M)$ for $i \sim n, 2 n$ where $\lambda(t)=1+r t^{n}$ and $r$ and $n$ are suitably chosen. In particular, $M$ is determined up to finitely many possibilities.

It is natural to conjecture that one could refine "up to finitely many possibilities" in Corollary 3 to "uniquely" by continuing the exact calculations in Proposition 2 to the cases $j=3,4, \ldots, j(k)$, for some $j(k)$, and/or by altering $\lambda(t)$. Indeed one might ask what would be the minimum value for $j(k)$. Unfortunately in adverse cases consideration of $j=3,4$ adds little to what is already known about $M$ from $j=1,2$. The algebraic complexity of the situation would seem to make this line of attack formidably difficult. Instead we adopt a different approach.

\section{UNIQUENESS OF THE SOLUTION OF THE COMBINATORIAL PROBLEM}

Let $p_{1}, \ldots, p_{s}$ be distinct odd primes, and let $r$ be a prime larger than the product $p_{1} p_{2} \cdots p_{s}$. Take $L$ such that $\|M\|<L$, and suppose $\lambda(t), n$ are chosen so that Lemma 1 pertains with $K=p_{1} \cdots p_{s}$.

Lemma 4. If $\lambda_{i}$ is divisible by $r$ for all $i \geq 1$, then $\lambda_{i}(M)$ and $\kappa_{i}(M)$ are divisible by $r$ for all $i$ in the range $1 \leq i \leq p_{1} \cdots p_{s} n+\frac{n}{2}$. 
Furthermore, for $i \sim p_{1} \cdots p_{s} n$

(i) if $M=\Sigma^{a} Y$, then

$$
\lambda_{i}(M)=\lambda_{i-a}(Y)
$$

(ii) if $M=\Omega \Sigma Y$, then

$$
\lambda_{i}(M) \equiv \lambda_{i}(Y) \quad \bmod r^{2}
$$

and

$$
r^{-1} \kappa_{i}(M) \equiv \sum_{e \mid p_{1} \cdots p_{s}} \mu(e) e^{-1} r^{-1} \lambda_{i / e}(Y) \bmod r
$$

(iii) if $M=\Omega^{b}(\Omega \Sigma Y), b>1$, then

$$
\kappa_{i}(M)=\kappa_{i+b}(\Omega \Sigma Y)
$$

and

$$
r^{-1} \lambda_{i}(M) \equiv \sum_{e \mid p_{1} \cdots p_{s}} e^{-1} r^{-1} \kappa_{i / e}(M) \bmod r .
$$

Proof. The first statement in the lemma is trivially true for $\|M\|=0$. Using (1.2)-(1.4) it is seen to be true for $Z=\Sigma^{a} Y$ and $Z=\Omega \Sigma Y$, if true for $Y$. Also by (1.7) $\kappa_{i}\left(\Omega^{b}(\Omega \Sigma) Y\right)$ is divisible by $r$. By $(1.6) \lambda_{i}\left(\Omega^{b}(\Omega \Sigma) Y\right)$ is divisible by $r$ for $1 \leq i \leq p_{1} \cdots p_{s} n+\frac{n}{2}$, since each binomial coefficient $\left(\begin{array}{l}\kappa \\ x\end{array}\right)$ and $\left(\begin{array}{c}\kappa+x-1 \\ x\end{array}\right)$ is divisible by $r$ for $1 \leq x \leq p_{1} \cdots p_{s}$. Hence, the first statement of Lemma 4 follows by induction on $\|M\|$.

For $i \sim p_{i} \cdots p_{s} n,(3.1)$ restates (1.2), (3.4) restates (1.7), and (3.2) follows from (1.3) and the first statement in this lemma. To prove (3.3) we note that in (1.4) we need only consider values of $d$ close to a multiple of $n$, since by Lemma $1 \lambda_{j}(Y)=0$ unless $j$ is close to a multiple of $n$. Setting $e=i / d$ we have $e \mid p_{1} \cdots p_{s}$, so $(-1)^{i+d}=(-1)^{d(e+1)}=1$ and, by the choice of $r$, $e \not \equiv 0 \bmod r$. As each $\lambda_{j}(Y)$ is divisible by $r$

$$
\frac{\lambda_{1}(Y)^{\beta_{1}} \lambda_{2}(Y)^{\beta_{2}} \cdots}{r\left(\beta_{1}+\beta_{2}+\cdots\right)} \equiv 0 \quad(\bmod r)
$$

whenever $\left(\beta_{1}+\beta_{2}+\cdots\right)>1$. Hence, to find $\kappa_{1}(Z) \bmod r^{2}$ we need only consider those terms on the right-hand side of (1.4) for which the $\beta_{j}$ are zero except for just one value of $j$, and, for this value of $j, \beta_{j}=1$ and $j(=d)$ divides $i$ and $e=i / j$ divides $p_{1} \cdots p_{s}$.

To prove (3.5) one notes that

$$
r^{-1}\left(\begin{array}{l}
\kappa \\
x
\end{array}\right) \equiv(-1)^{x-1} x^{-1} r^{-1} \kappa \quad(\bmod r)
$$

and

$$
r^{-1}\left(\begin{array}{c}
\kappa+x-1 \\
x
\end{array}\right) \equiv x^{-1} r^{-1} \kappa \quad(\bmod r)
$$

when $x \leq p_{1} \cdots p_{s}<r$ and $\kappa$ is divisible by $r$. Hence, expanding the righthand side of (1.6), one obtains (3.5).

We now take $\lambda(t)=1+r t^{n}$ in the above. Lemma 4 provides the inductive step in the proof of 
Proposition 5. Let $\lambda(t)=1+r t^{n}$ and $r, p_{1}, \ldots, p_{s}, n$ be constrained to be chosen as described above. Then

$$
\sum_{i \sim p_{1} \cdots p_{s} n} r^{-1} \lambda_{i}(M) t^{i} \equiv\left(p_{1} \cdots p_{s}\right)^{-1} \sum \sigma(\mathbf{x}) t^{p_{1} \cdots p_{s} n+S(\mathbf{x})} \quad(\bmod r)
$$

where the sum on the right-hand side is taken over all those cascading $2 k$-tuples that satisfy:

(i) $x_{1}=p_{1} \cdots p_{s}$,

(ii) $x_{2 j-1}=x_{2 j}=x_{2 j+1}$ if $b_{j}=0$ and $j<k$,

(iii) $x_{2 k-1}=x_{2 k}=1$ if $b_{k}=0$.

Here $\sigma(\mathbf{x})=(-1)^{\nu_{1}+\nu_{2}+\cdots+\nu_{2 k}}$ where $x_{i}$ is the product of precisely $\nu_{i}$ of the primes $p_{1}, \ldots, p_{s}$.

As a demonstration of how Proposition 5 may be exploited we explain how $M$ may be recovered from $\lambda(M, t)$ when $M$ is a positive word. By Corollary 3 we already know $k$. Take $s=2 k$ in Proposition 5 . The $2 k$-tuple $\mathbf{x}=$ $\left(p_{1} p_{2} \cdots p_{2 k}, p_{1} \cdots p_{2 k-1}, \ldots, p_{1} p_{2}, p_{1}\right)$ is the only cascading $2 k$-tuple that contributes a term $t^{i}$ where $i$ has the form $\mu_{0}=\mu_{1} p_{1}+\mu_{2} p_{1} p_{2}+\cdots+\mu_{2 k} p_{1} \cdots p_{2 k}$ with all $\mu_{i}$ nonzero. The freedom of choice for $p_{1}, \ldots, p_{2 k}$ allows one to distinguish this value of $i$ (from the other powers coming from cascading $2 k$-tuples) and to deduce the values of $\mu_{0}, \ldots, \mu_{2 k}$ (which are independent of the choice) uniquely. $M$ is recovered since $a_{k+1}=\mu_{0}, b_{k}=-\mu_{1}$, $a_{k}=\mu_{2}, \ldots, b_{1}=-\mu_{2 k-1}, a_{1}=\mu_{2 k}-n$.

This completes the proof of our theorem.

Remark. The above argument distinguishes a positive word from any other (positive or nonpositive) word. An analogous argument distinguishes between two nonpositive words (not already distinguished by Corollary 3 ), provided that one word cannot be converted into the other by replacing subwords $\Sigma(\Omega \Sigma)^{r} \Sigma$ by $\Sigma(\Omega \Sigma)^{s} \Sigma$ (for distinct $r, s \geq 0$ ) or subwords $\Omega(\Omega \Sigma)^{r} \Omega$ by $\Omega(\Omega \Sigma)^{s} \Omega$ (for distinct $r, s \geq 1)$.

\section{APPENDIX}

Proof of Lemma 1. We verify by induction on $L$ that one can take

$$
\begin{gathered}
C(K, L, R)=\left(K R+2 R\left(u+2 L^{2}\right) K^{L}\right)^{K^{2 L}}, \\
D(K, L)=\left(u+2 L^{2}\right) K^{L}, \\
N(K, L)=2\left(u+2 L^{2}\right)(K+1)^{L}+2 L(K+1) .
\end{gathered}
$$

For each word $M$ define the coefficient maximum $c_{M}$ and the dispersion $d_{M}$ of $\lambda(M, t)$ by.

$$
\begin{gathered}
c_{M}=\sup \lambda_{i}(M), \\
d_{M}=\sup \left\{\left|i-\left[\frac{i}{n}+\frac{1}{2}\right] n\right|: \lambda_{i}(M) \neq 0\right\}
\end{gathered}
$$

where the suprema are taken over all $i$ satisfying $i \leq K n+\frac{n}{2}$. The values of $c_{M}$ and $d_{M}$ of course depend on $M, K, n$, and $\lambda(t)$, but we shall show that if $n>N(K, L)$ then

$$
c_{M} \leq C(K, L, R)
$$


and

$$
d_{M} \leq D(K, L) .
$$

For $L=1$ the only possibility for $M$ is the empty word $I$ for which $c_{I}<R$, $d_{I} \leq u$, and hence (4.6) and (4.7) hold for all $M$ satisfying $\|M\|<L$ (and every $K, n, \lambda(t))$. For $Z \in \mathscr{M}$ with $\|Z\|=L$ we now check Cases $1,2,3$ detailed in $\S 2$.

Case 1. If $Z=\Sigma^{a} Y$, where $a \geq 1$, then by (1.2) $c_{Z} \leq c_{Y}$ and $d_{Z} \leq d_{Y}+a \leq$ $d_{Y}+L$. When $n>N(K, L)$, then certainly $n>N(K, L-1)$ and from (4.6) and (4.7) for $Y$

$$
c_{Z} \leq c_{Y} \leq C(K, L-1, R)<C(K, L, R)
$$

and

$$
d_{Z} \leq D(K, L-1)+L<D(K, L) .
$$

Case 2. If $Z=\Omega \Sigma Y$ and $n>N(K, L)$, then by (4.2) and (4.3) $n>$ $2(K+1) D(K, L-1)$. It follows that for $i \leq K n+\frac{n}{2}$ each contribution to $\lambda_{i}(Z)$ in (1.3) is a product of at most $K$ of the $\lambda_{j}(Y)$ (with $\left.j \leq i\right)$. Thus

$$
d_{Z} \leq K d_{Y} \leq K D(K, L-1) \leq D(K, L) \text {. }
$$

Note that by (4.5) the number of positive values of $i$ such that $i \leq K n+\frac{n}{2}$ and $\lambda_{i}(M) \neq 0$ is not greater than $K+2 K d_{Y}$, so by (1.3)

$$
\begin{aligned}
c_{Z} & \leq\left(K+2 K d_{Y}\right)^{K} c_{Y}^{K} \\
& \leq\left(K+2 K\left[u+2(L-2)^{2}\right] K^{L-2}\right)^{K}\left(K r+2 r\left[u+2(L-2)^{2}\right] K^{L-2}\right)^{K^{2(L-2)+1}} \\
& \leq C(K, L, R) .
\end{aligned}
$$

Case 3. Suppose $Z=\Omega^{b}(\Omega \Sigma Y), b \geq 1, n>N(K, L)$. Denote the coefficient maximum and dispersion of $\kappa(M, t)$ by $c_{M}^{\prime}, d_{M}^{\prime}$, i.e., $c_{M}^{\prime}=\sup \kappa_{i}(M)$, $d_{M}^{\prime}=\left\{\left|i-\left[\frac{i}{n}+\frac{1}{2}\right] n\right|: \kappa_{i}(M) \neq 0\right\}$, where the suprema are taken over all $i$ satisfying $i \leq K n+\frac{n}{2}$. Trivially $c_{M}^{\prime} \leq c_{M}, d_{M}^{\prime} \leq d_{M}$. As $n>N(K, L)$ we have

$$
n>2 D(K+1, L)+2 L \geq 2 D(K+1, L-1)+2 b .
$$

Thus if $\lambda_{i}(\Omega \Sigma Y) \neq 0$ and $(K+1) n$ is the multiple of $n$ closest to $i$, then $(K+1) n$ is the multiple of $n$ closest to $i-b$. Hence,

$$
d_{Z}^{\prime} \leq d_{\Omega \Sigma Y}^{\prime}+b \leq d_{\Omega \Sigma Y}^{\prime}+L \leq D(K, L-1)+L
$$

and

$$
c_{Z}^{\prime} \leq c_{\Omega \Sigma Y}^{\prime} \leq c_{\Omega \Sigma Y} \leq C(K, L-1, R) .
$$

As $n>N(K, L)>2(K+1) D(K, L-1)+2 L(K+1) \geq 2(K+1) d_{Z}^{\prime}$ it follows that in expanding the right-hand side of (1.6) using binomial series a term $t^{j}$ with $\left[\frac{j}{n}+\frac{1}{2}\right]>K$ is obtained if (in total) more than $K$ of the $t^{i}$ are multiplied together. Thus

$$
d_{Z} \leq K d_{Z}^{\prime}<K(D(K, L-1)+L)<D(K, L) .
$$

Since $\left(c_{Z}^{\prime}+K\right)^{j}$ exceeds the binomial coefficients

$$
\left(\begin{array}{c}
\kappa_{i}(Z) \\
j
\end{array}\right) \text { and }\left(\begin{array}{c}
\kappa_{i}(Z)+K \\
j
\end{array}\right)
$$


from (1.6) we have

$$
\begin{aligned}
c_{Z} & \leq\left(K+2 K d_{Z}^{\prime}\right)^{K}\left(c_{Z}^{\prime}+K\right)^{K} \leq(K+2 D(K, L))^{K}(C(K, L-1, R)+K)^{K} \\
& \leq\left(K+2\left(u+2 L^{2}\right) K^{L}\right)^{K}\left[\left(K R+2 R\left[u+2(L-1)^{2}\right] K^{L-1}\right)^{K^{2 L-2}}+K\right]^{K} \\
& \leq\left(K+2\left(u+2 L^{2}\right) K^{L}\right)^{K}\left[\left(K R+2 R\left[u+2 L^{2}\right] K^{L-1}\right)^{K^{2 L-2}}\right]^{K} \\
& \leq\left(K R+2 R\left(u+2 L^{2}\right) K^{L}\right)^{K+K^{2 L-1}} \leq C(K, L, R) .
\end{aligned}
$$

Proof of Proposition 2. Use induction on $L$. When $L=1$ the proposition holds trivially as the only possibility for $M$ is $M=I$. We first establish the particular statements (i), (ii) by the following calculations. Suppose the inductive hypothesis that, for $\|M\|<L$,

$$
\lambda(M, t)=1+r t^{n+C(M)}+\sum_{\mathbf{x}} c(M)(\mathbf{x}) t^{2 n+S(M)(\mathbf{x})}+\cdots,
$$

where the higher-order terms are suppressed in the dots.

Case 1. If $Z=\Sigma^{a} M$, then by (1.2)

$$
\begin{aligned}
\lambda(Z, t) & =1+r t^{n+C(M)+a}+\sum_{\mathbf{x}} c(M)(\mathbf{x}) t^{2 n+S(M)(\mathbf{x})+a}+\cdots \\
& =1+r t^{n+C(Z)}+\sum_{\mathbf{x}} c(Z)(\mathbf{x}) t^{2 n+S(Z)(\mathbf{x})}+\cdots
\end{aligned}
$$

since $C(Z)=C(M)+a, c(Z)(\mathbf{x})=c(M)(\mathbf{x}), S(Z)(\mathbf{x})=S(M)(\mathbf{x})+a$.

Case 2. If $Z=\Omega \Sigma M$, then by (1.3)

$$
\lambda(Z, t)=1+r t^{n+C(M)}+\sum_{\mathbf{x}} c(M)(\mathbf{x}) t^{2 n+S(M)(\mathbf{x})}+r^{2} t^{2 n+2 C(M)}+\cdots
$$

We associate to each $2 k$-tuple $\mathbf{x}$ the $(2 k+2)$-tuple $\mathbf{y}=\left(x_{1}, \ldots, x_{2 k}, 1,1\right)$, and since $b_{k+1}=a_{k+2}=0$ in the expression (1.1) for $Z$, we have $S(M)(\mathbf{x})=$ $S(Z)(\mathbf{y}), c(M)(\mathbf{x})=c(Z)(\mathbf{y}), C(M)=C(Z)$. The term $r^{2} t^{2 n+2 C(M)}$ can be viewed as supplying the contributions $c(Z)(\mathbf{y}) t^{2 n+S(M)(y)}$ for the $(2 k+2)$-tuples $\mathbf{y}=(2, \ldots, 2,2,1)$ and $\mathbf{y}=(2,2, \ldots, 2,2)$ to obtain now the formula (4.8) for $Z$.

Case 3. If $Z=\Omega^{b}(\Omega \Sigma M)$, where $b>0$, then by (1.4)

$$
\begin{aligned}
\kappa(\Omega \Sigma M, t)= & 1+r t^{n+C(M)}+\frac{r}{2}\left(r-(-1)^{n+C(M)}\right) t^{2 n+2 C(M)} \\
& +\sum_{\mathbf{x}} c(M)(\mathbf{x}) t^{2 n+S(M)(\mathbf{x})}+\cdots
\end{aligned}
$$

By (1.6)

$$
\begin{aligned}
\lambda(Z, t)= & +r t^{n+C(M)-b}+\frac{r}{2}\left(r+(-1)^{n+C(M)-b}\right) t^{2(n+C(M)-b)} \\
& +\frac{r}{2}\left(r-(-1)^{n+C(M)}\right) t^{2 n+2 C(M)-b} \\
& +\sum_{\mathbf{x}} c(M)(\mathbf{x}) t^{2 n+S(M)(\mathbf{x})-b}+\cdots
\end{aligned}
$$

For any $2 k$-tuple $\mathbf{x}$ we have $S(Z)(\mathbf{x}, 1,1)=S(M)(\mathbf{x})-b$ and $c(Z)(\mathbf{x}, 1,1)=$ $c(M)(\mathbf{x})$. Also $C(Z)=C(M)-b$. Thus (4.9) yields the formula (4.8) for $Z$, where the summation over $2 k$-tuples $\mathbf{x}$ corresponds to summation over 
the $(2 k+2)$-tuples of the form $(\mathbf{x}, 1,1)$ and the two previous terms give the contributions from the $(2 k+2)$-tuples $(2,2, \ldots, 2)$ and $(2,2, \ldots, 2,1)$. This completes the proof of (i) and (ii).

Consideration of the above Cases 1,2,3 also yields the inductive step of a proof of the other claim in Proposition 2. In Case $1 \lambda_{i}(Z)=\lambda_{i-a}(M)$ and $S(Z)(\mathbf{x})=S(M)(\mathbf{x})+a$, hence the result. In Case 2 , by $(1.3) \lambda_{i}(Z)=0$ unless $i=i_{1}+i_{2}+\cdots$ and $\lambda_{i_{1}}(M), \lambda_{i_{2}}(M), \ldots$ are all nonzero. Thus

$$
i=j_{1} n+S(M)\left(\mathbf{x}^{(1)}\right)+j_{2} n+S(M)\left(\mathbf{x}^{(2)}\right)+\cdots+j_{q} n+S(M)\left(\mathbf{x}^{(q)}\right),
$$

where $\mathbf{x}^{(1)}, \ldots, \mathbf{x}^{(q)}$ are descending $2 k$-tuples (not necessarily distinct) with $x_{1}^{(1)}=j_{1}, \ldots, x_{1}^{(q)}=j_{q}$. So $i=j n+S(M)(\mathbf{x})+q a_{k+1}$, where $j=j_{1}+$ $\cdots+j_{q}$ and $\mathbf{x}=\mathbf{x}^{(1)}+\cdots+\mathbf{x}^{(q)}$, and where $x_{1}=j$ and $x_{2 k} \geq q$. Thus $i=j n+S(Z)(\mathbf{y})$, where $\mathbf{y}$ is the $(2 k+2)$-tuple $\left(x_{1}, \ldots, x_{2 k}, q, 1\right)$. In Case 3 we know that $\kappa_{i}(\Omega \Sigma M) \leq \lambda_{i}(\Omega \Sigma M)$ and hence that $\kappa_{i}(\Omega M)$ vanishes unless $i=j n+S(\Omega \Sigma M)(\mathbf{y})$ for some descending $(2 k+2)$-tuple $\mathbf{y}$ with $y_{1}=j$ and $y_{2 k+2}=1$. Since $S(Z)(\mathbf{y})=S(\Omega \Sigma M)(\mathbf{y})-b$, we have that $\kappa_{i}(Z)=0$ unless $i=i_{1}+\cdots+i_{q}$, where $i_{1}, \ldots, i_{q}$ are not necessarily distinct but each $i_{t}$ has the form $j_{t} n+S(Z)\left(\mathbf{y}^{(t)}\right)$. Since $a_{k+2}=0$ in the expression (1.1) for $Z$, each $i$ has the form $j n+S(Z)(\mathbf{y})$ where $\mathbf{y}=\mathbf{y}^{(1)}+\cdots+\mathbf{y}^{(t)}$ and $j=y_{1}$.

It is perhaps worth remarking that, for sufficiently large $r, \lambda_{i}(M)$ is nonzero if and only if $i$ has the form described.

\section{REFERENCES}

1. D. J. Anick, The computation of rational homotopy groups is \#P-hard, Computers in geometry and topology (Chicago IL, 1986), Lecture Notes in Pure and Appl. Math., vol. 114, Dekker, New York, 1989, pp. 1-56.

2. I. M. James, Reduced product spaces, Ann. of Math. (2) 62 (1955), 170-197.

3. T. Miller and J. Neisendorfer, Formal and coformal spaces, Illinois J. of Math. 22 (1978), 565-580.

4. E. Witt, Treue Darstellung Liescher Ringe, J. Reine Angew. Math. 177 (1937), 152-160.

Department of Mathematics, Edinburgh University, James Clerk Maxwell Building, The KING's BuIldings, EdINBURGH EH9 3JZ, Scotland

E-mail address: m.eggar@ed.ac.uk 